\title{
Guided Wave Inspection of Bars in Reinforced-Concrete Beams Using Surface-Mounted Vibration Sensors
}

\author{
Evelyne El Masri *, Timothy Waters and Neil Ferguson \\ Institute of Sound and Vibration Research, University of Southampton, Hampshire SO17 1BJ, UK; \\ T.P.Waters@soton.ac.uk (T.W.); nsf@isvr.soton.ac.uk (N.F.) \\ * Correspondence: eem1c18@soton.ac.uk
}

Received: 25 August 2020; Accepted: 23 September 2020; Published: 27 September 2020

check for updates

\begin{abstract}
Steel reinforcement bars (rebars) in concrete structures are inaccessible and not conducive to many inspection methods. This paper proposes a non-invasive technique based on guided waves for detecting localised abnormalities in rebars embedded in concrete beams. The technique is predicated on previously published observations that guided waves are strongly reflected by discontinuities at the frequency at which they begin to propagate, i.e., at cut-on. The reflection coefficient at cut-on is estimated using a simple wave decomposition in which a near-zero wavenumber value is assumed. A simulated study is first carried out to evaluate the technique on a concrete beam featuring four rebars. The wave finite element approach is adopted to model two uniform beams which are coupled via a short, damaged section modelled in conventional finite element analysis. Estimated reflection coefficients arising from the discontinuity are close to the true values at cut-on and independent of frequency elsewhere, so that no prior knowledge of cut-on frequencies is required. Three steel-reinforced concrete beams were fabricated-one uniform and two with localised rebar damage - and reflection coefficients were estimated from measured transfer functions. As predicted, abrupt deviations in the reflection coefficient occurred at cut-on frequencies for both damaged beams.
\end{abstract}

Keywords: guided waves; reinforced concrete; damage; non-destructive evaluation

\section{Introduction}

Damage in reinforced concrete $(\mathrm{RC})$ is often initiated through corrosion of the embedded steel reinforcement bars (rebars). Structural health-monitoring methods to detect corrosion are important to identify interior degradation at a sufficiently early stage to take remedial action. In practice, significant reliance is still placed on visual inspection, which is limited to surface flaws resulting from severe internal damage.

Of the many instrumented approaches to periodic inspection or monitoring of steel reinforcement bars, wave-based methods are most prevalent in the research literature. Techniques may be classified as either passive or active. Passive techniques use ultrasonic sensors to 'listen' to acoustic emissions generated by micro-crack growth under operational loading. Active methods excite guided waves and infer the presence of damage from changes in their propagation characteristics. Sharma et al. compared passive and active approaches for the detection of rebar corrosion [1,2]. In their study, acoustic emission was found to be more effective for monitoring initial corrosion and guided waves were preferable for identifying the nature of the damage.

This paper is concerned with active techniques, which were comprehensively reviewed in 2013 by Lei and Zheng [3]. In almost all cases in the reviewed literature, and since, an actuator and sensor pair are attached directly to opposing ends of a steel rebar in a pitch-transmission configuration. Where the rebar is embedded in concrete this is enabled in a laboratory setting by leaving the ends exposed, but in practical applications transducers must be embedded in the composite beam during 
construction. One commonly advocated approach to detecting corrosion has been to measure the strength of longitudinal [4,5] and/or flexural [6] wave transmission along a rebar. A significant limitation is that the signal strength may either increase or reduce with corrosion. This depends on whether the predominant effect of the corrosion is to couple more strongly with the concrete through increased surface roughness or reduced coupling through eventual delamination and separation. Furthermore, the strength of the signal is influenced by the bonding of the transducers. Sharma and Mukherjee proposed combining the pulse-transmission approach with pulse-echo [7]. Simulations on a single rebar embedded in concrete showed a significant reflected pulse, carried by a high order longitudinal mode, when a $40-60 \%$ notch was introduced to the rebar. A similar numerical study, exploiting the fundamental longitudinal mode, employed wavelet analysis to help extract a weak reflection due to a notch from a multitude of other reflections [8]. An alternative approach is to identify changes in wave dispersion due to damage by monitoring the time-of-flight of a prevailing wave type; this is independent of transducer bonding. Miller et al. observed that when a rebar is loaded in compression due to transverse loading of the composite beam then rebar corrosion causes slippage with respect to the concrete which reduces axial stress in the rebar and hence increases wave speed [9]. Amjad et al. [10] measured the time-of-flight of longitudinal waves in corroded steel rebars in vacuo, concluding that the $\mathrm{L}(0,1)$ mode was highly sensitive to corrosion level. Majhi et al. observed from their measurements on a steel bar that spectral content was more sensitive to corrosion than time-of-flight [11]. Time-frequency analysis was performed on response data using the S-transform. Correlation to simulated dispersion curves showed a shift in spectral contribution with corrosion from higher- to lower-order modes. Preliminary results were also presented for an instrumented, embedded rebar. Zima and Kędra used time-of-flight of longitudinal waves in embedded rebars to estimate the length of debonding [12]. No baseline data were used, but dispersion curves were required from an accurate model in order to determine the effects of debonding length on wave velocity. Climent et al. correlated harmonic distortion of the response to non-linearities arising from crack growth using surface mounted ultrasonic transducers [13]. Crack growth was successfully identified in a laboratory specimen with higher than normal corrosion rates.

An alternative methodology, which allows for inspection of embedded rebars from near-surface measurements, is ultrasonic tomography. In contrast to the aforementioned guided wave techniques, an ultrasonic transmitter generates through-cross-section waves, which are received by an array of transducers on the opposing side. The group velocity distribution is determined, typically from time of flight estimates, from which abnormalities such as debonding and inclusions can be successfully visualised [14]. Inspection of a length of beam requires the technique to be applied repeatedly at different stations.

In this paper, a guided wave-based technique is proposed with the express aim of detecting rebar damage from transducers mounted on the concrete surface and remotely from the damage site. It is intended for periodic inspection rather than continuous monitoring so that it can be deployed retrospectively to structures without embedded sensors. In contrast to most guided wave techniques in the literature which are ultrasonic and formulated in the time domain, the proposed method is based on transfer functions at, typically, audio frequencies. A simplified wave decomposition is used to estimate reflection from defects, which is found to be measurably large at wave cut-on.

This paper is structured as follows. Section 2 outlines the wave decomposition method by which reflection from discontinuities in rebars can be quantified. A wave finite element model of a reinforced concrete beam, reported in detail in [15], is briefly outlined and simulations of wave reflection from a defect in a rebar are presented for several idealised boundary conditions. In Section 2, experimental samples of steel-reinforced concrete beams are fabricated with a localised loss of diameter in one rebar. Instrumented hammer tests are conducted to obtain transfer functions on the exposed beam surfaces. Subsequently inferred reflection coefficients clearly indicate the existence of damage. Potential limitations of the technique are discussed in the Conclusions section, together with future work required to explore its domain of applicability. 


\section{Damage Identification Using Guided Waves}

The overall goal of this work is to propose and validate a damage-detection algorithm for steel-reinforced concrete beams that does not require response measurements on inaccessible reinforcements. In principle, localised damage to reinforcements may induce reflection of guided waves and this may be detectable from surface vibration measurements. However, accurate estimation of reflection coefficients of multiple wave types is impractical. The problem is made tractable here by noting that guided waves are strongly reflected close to their cut-on frequencies [15] at which the wavenumber of the predominant wave is close to zero. The phenomenon arises when a wave can propagate in the undamaged section of the waveguide and not the damaged section, or vice versa. Estimation of wave amplitudes is simplified to a single incident and reflected wave of near-zero wavenumber for which a standard wave amplitude decomposition (WAD) is suited [16].

In this section, the WAD method is briefly reviewed and applied specifically to the estimation of a single reflection coefficient at wave cut-on. The accuracy of the method is then assessed on a simulated example. Infinite RC beams, modelled using the wave finite element (WFE) method, are coupled via a short finite element model of the same or similar cross section, namely with and without localised loss of diameter in one rebar. The analysis is then extended to a finite beam with perfectly reflecting boundaries.

\subsection{Damage Detection Using Wave Amplitude Decomposition}

The WAD method enables amplitudes of positive and negative going waves to be estimated at the mid-point of an array of transducer measurements. The wave amplitude vector $\mathbf{A}$ at a specific frequency can be related to complex amplitudes $\mathbf{W}$, or equivalently frequency response functions (FRFs), pertaining to displacement, velocity or acceleration, at positions $x_{1}$ to $x_{n}$ by,

$$
\mathbf{W}=\Lambda \mathbf{A}
$$

where $\mathbf{W}=\left\{W\left(x_{1}\right), \cdots, W\left(x_{n}\right)\right\}^{T}, \mathbf{A}=\left\{\mathbf{A}^{+}, \mathbf{A}^{-}\right\}^{T}$ represents $m$ positive and $m$ negative going wave amplitudes, $\Lambda$ is a propagation matrix,

$$
\boldsymbol{\Lambda}=\left[\begin{array}{cccccc}
e^{-i k_{1} x_{1}} & \cdots & e^{-i k_{m} x_{1}} & e^{i k_{1} x_{1}} & \cdots & e^{i k_{m} x_{1}} \\
e^{-i k_{1} x_{2}} & \cdots & e^{-i k_{m} x_{2}} & e^{i k_{1} x_{2}} & \cdots & e^{i k_{m} x_{2}} \\
\vdots & \vdots & \vdots & \vdots & \cdots & \vdots \\
e^{-i k_{1} x_{n}} & \cdots & e^{-i k_{m} x_{n}} & e^{i k_{1} x_{n}} & \cdots & e^{i k_{m} x_{n}}
\end{array}\right]
$$

and $\pm k_{i}$ are the wavenumbers of the $i$ th positive and negative-going waves. In general, one needs to include all waves that contribute significantly to the observed response and prior knowledge of their dispersion relations is required to evaluate the propagation matrix. Evanescent waves can often be neglected by placing the transducer array in the far field of boundaries and the excitation point. Typically, there are more transducers than unknown wave amplitudes such that a least squares solution is found using the pseudo-inverse,

$$
\mathbf{A}=\left(\boldsymbol{\Lambda}^{H} \boldsymbol{\Lambda}\right)^{-1} \boldsymbol{\Lambda}^{H} \mathbf{W}
$$

where superscript $H$ denotes conjugate transpose. The transducer spacing should be less than half the shortest wavelength in the frequency range of interest to avoid ill-conditioning of the pseudo-inverse. Conditioning also deteriorates as the number of unknown wave amplitudes increases. Additional studies related to the location and spacing of transducers, and associated matrix conditioning, are reported in [16].

The method is then applied to the specific case of estimating reflection coefficients from localised damage in a waveguide. It is assumed, as reported by [15], that strong reflection occurs predominantly at wave cut-on and that, since cut-on is associated with a resonance of the cross-section, the response 
is dominated by the wave cutting on. The problem is reduced to estimating a single incident and reflected wave, their ratio being an estimate of the reflection coefficient for that wave at its cut-on. The propagation matrix reduces to two columns,

$$
\boldsymbol{\Lambda}=\left[\begin{array}{cc}
e^{-i k_{s} x_{1}} & e^{i k_{s} x_{1}} \\
\vdots & \vdots \\
e^{-i k_{s} x_{n}} & e^{i k_{s} x_{n}}
\end{array}\right]
$$

where $k_{s}$ is small, because the wave is assumed to have just cut-on, but not zero to avoid singularity of $\Lambda$. A real value of $0.1 \mathrm{rad} / \mathrm{m}$ is chosen for all simulations presented in this paper. Investigations regarding usage of other values of wavenumber (smaller or complex) are presented in [17].

\subsection{Wave Model of Reinforced Concrete (RC) Beam}

Simulations were performed using a WFE model of a RC beam. The WFE approach computes the free wave propagation in a waveguide by modelling a short section in conventional finite elements. Periodic boundary conditions are then imposed such that the nodal displacements and forces on one side of the section differ from those on the other side by a phase shift corresponding to the product of wavenumber and section length. At each frequency, an eigen problem is obtained for all possible wavenumbers and their corresponding wave mode shapes. Forced response calculations can be performed by applying equilibrium of forces and displacements at the forcing point. Discontinuities, such as damage, in an otherwise uniform waveguide can be modelled using the WFE-FE-WFE coupling approach [18]. The undamaged lengths are modelled in WFE whilst the intermediate damaged part, which might be geometrically complex and non-uniform, is modelled in FE. The components are then assembled by application of equilibrium of forces and displacements. Scattering coefficients due to the presence of the damage can be computed as reported in [15].

The RC beam example in this paper had four rebars, one of which was reduced in diameter to simplistically represent damage. All material properties were as reported in [15] and $0.4 \%$ hysteretic damping was included. The finite element model of the section is shown in Figure 1.

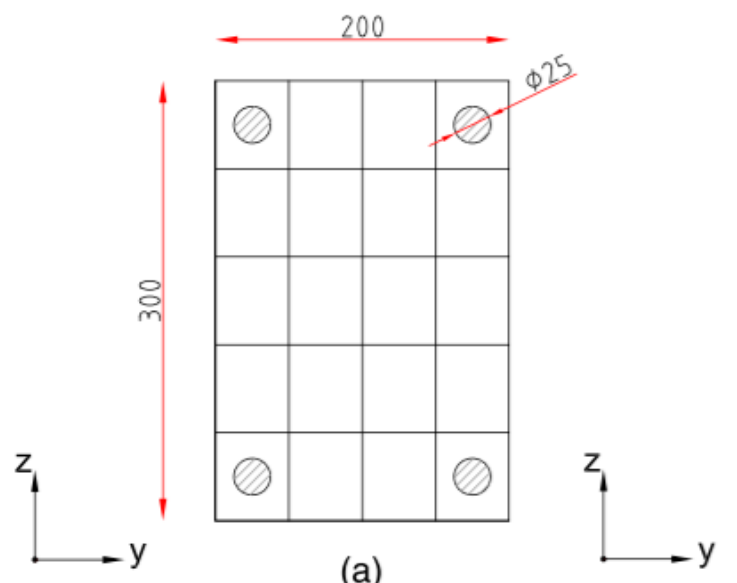

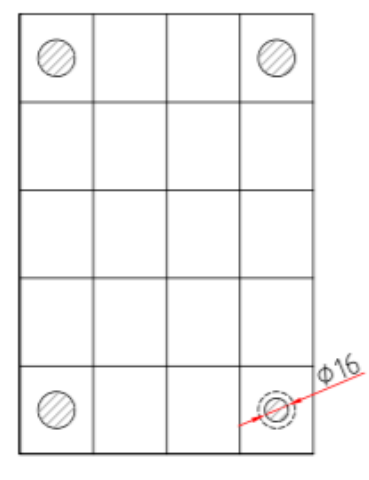

(b)

Figure 1. Finite element (FE) mesh of reinforced concrete (RC) sections: (a) undamaged and (b) damaged with a rebar diameter reduction of $40 \%$. Dimensions are in $\mathrm{mm}$.

The RC section was modelled in Ansys Mechanical ADPL 2014 (Ansys, Canonsburg, Pennsylvania, USA) using 16 SOLID65 elements. The horizontal rebars were modelled via the embedded approach using REINF264 elements. The length of the segment in the $\mathrm{x}$ direction, $\Delta$ was set equal to $0.01 \mathrm{~m}$. All material properties are presented in Table 1. 
Table 1. FE material properties for concrete and steel.

\begin{tabular}{ccc}
\hline Material Properties & Concrete & Steel \\
\hline Young's modulus $E(\mathrm{~Pa})$ & $40 \times 10^{9}$ & $200 \times 10^{9}$ \\
Poisson ratio $v$ & 0.18 & 0.3 \\
Density $\rho\left(\mathrm{kg} / \mathrm{m}^{3}\right)$ & 2400 & 7850 \\
\hline
\end{tabular}

\subsection{Wave Amplitude Estimation in an Infinite Beam without Damage}

The simplified WAD approach outlined in Section 2.1 to estimate wave amplitudes at wave cut-on was first evaluated on an infinite undamaged beam. A harmonic force was applied in the transverse $(\mathrm{z})$ direction, on the centreline. Only positive-going waves existed to the right of the excitation, hence the propagation matrix $\Lambda$ was reduced to a single column. FRFs were computed in the z-direction at an array of four equally spaced positions along the beam's centreline, at 0.30 to $0.45 \mathrm{~m}$ from the excitation. Figure 2 shows the single-wave amplitude estimate at each frequency in a range spanning multiple cut-on frequencies. Also plotted are the known amplitudes for lightly attenuated waves, chosen here as $\operatorname{Im}\left(k_{i}\right)<5 \mathrm{rad} / \mathrm{m}$, from the full WFE solution. Away from cut-on, where the estimate is meaningless, there is only a modest resemblance to any of the actual wave amplitudes. At the cut-on frequencies, particularly those that are well separated $(8700,11,700,12,500 \mathrm{~Hz})$, the estimate aligns reasonably well with the amplitude of the dominant wave. At these particular frequencies, evanescent waves start to propagate and resonances occur featuring elastic deformation of the cross section. The associated mode shapes are reported in [15].

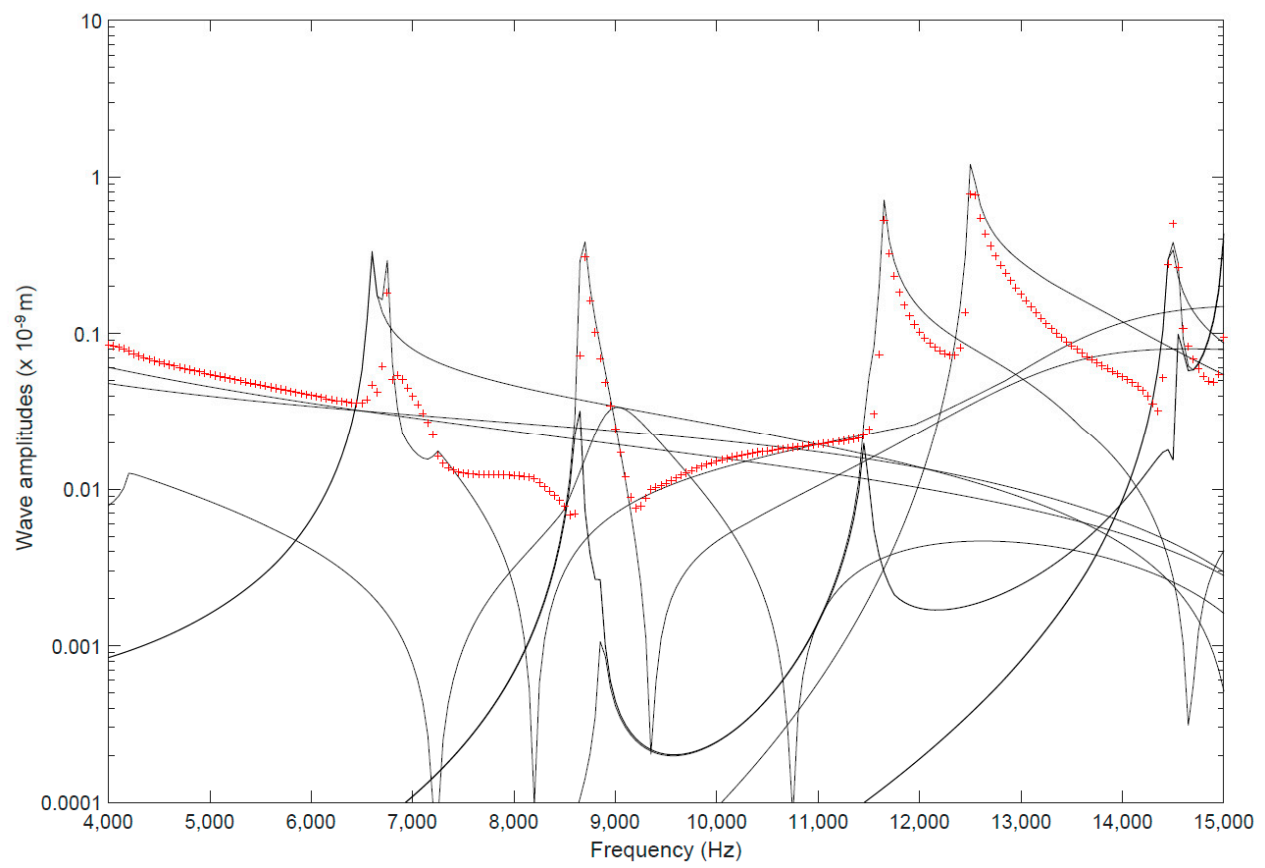

Figure 2. Wave amplitudes of lightly attenuated waves in an infinite undamaged RC beam. Wave finite element (WFE) solution (-), single-wave wave amplitude decomposition (WAD) estimate using $k_{s}=0.1$ $\mathrm{rad} / \mathrm{m}(+)$.

If the technique proves equally able to resolve both positive and negative going waves, then it may be used to infer reflection coefficients arising from damage.

\subsection{Wave Amplitude Estimation in an Infinite Beam with Damage}

Figure 3 shows two undamaged waveguides modelled in WFE, as in Section 2.2, joined to a damaged section modelled in conventional FE. The damaged section is identical to the undamaged 
sections except that one rebar has been reduced in diameter by 40\%, as shown previously in Figure 1. Positive going waves $\mathbf{a}^{+}$are incident on the damaged section from waveguide 1 giving rise to reflected and transmitted waves $\mathbf{a}^{-}$and $\mathbf{b}^{+}$. A two-wave WAD estimation was performed to obtain the predominant incident wave and reflected wave in waveguide 1, and these are overlaid in Figure 4 with the known wave amplitudes of all lightly attenuated waves obtained from the WFE solution. Both the incident and reflected waves are large in amplitude at cut-on and are captured accurately by the two-wave WAD estimate. The reflected and incident waves differ in magnitude only at cut-on, due to damage. Elsewhere the amplitude estimates for the incident and reflected waves are small and almost identical, which is more apparent on a logarithmic $y$-axis, see Figure 5 . The estimates are devoid of physical meaning, owing to the incorrect assumption that only one wave type dominates the response and the erroneous, small value of wavenumber attributed to it. However, since their values are the same, the reflection coefficient defined by their ratio is unity everywhere except at cut-on frequencies. Consequently, no prior knowledge of cut-on frequencies is required in order to discriminate between frequencies at which the reflection coefficient should be noted or discarded.

\subsection{Damage Detection in a Damaged Beam with Perfectly Reflecting Boundaries}

In practical applications, strong wave reflections occur over broad frequency bands due to the presence of boundaries and this might contaminate any estimates of reflection from damage. Perfectly reflecting boundaries are now added to the infinite beam model described in Section 2.4 to introduce the effect of additional scatterers whilst omitting the further complication of wave mode conversion at the boundaries. The wave field was determined from propagation matrices and reflection matrices associated with the damaged section and the boundaries.

Figure 6 shows a schematic of the WFE-FE-WFE beam model which is $2 \mathrm{~m}$ in length. The excitation force is to the left of a short, damaged section and an array of four equally spaced response positions is placed between the excitation and the damage. The damage is simulated by loss of diameter in one rebar. A two-wave WAD estimate was obtained from the four simulated FRFs.

Figure 7 shows the ratio of the reflected to incident wave amplitudes for the three cases of $0 \%$, $40 \%$ and $60 \%$ loss of thickness in one rebar, respectively. In the undamaged case, there is apparent total reflection at all frequencies including at cut-on where the reflection is due to the right end boundary. Away from cut-on the estimate is erroneous for the same reasons as for the infinite beam, and consistency with the boundary conditions is coincidental. When damage is imposed then the ratio of wave amplitudes is less than unity just at the cut-on frequencies of 8700, 11,700 and 12,500 $\mathrm{Hz}$. The values of this ratio agree approximately with the true magnitudes of the reflection coefficients as predicted by the model and presented in earlier work [15]. The values are compared in Table 2.

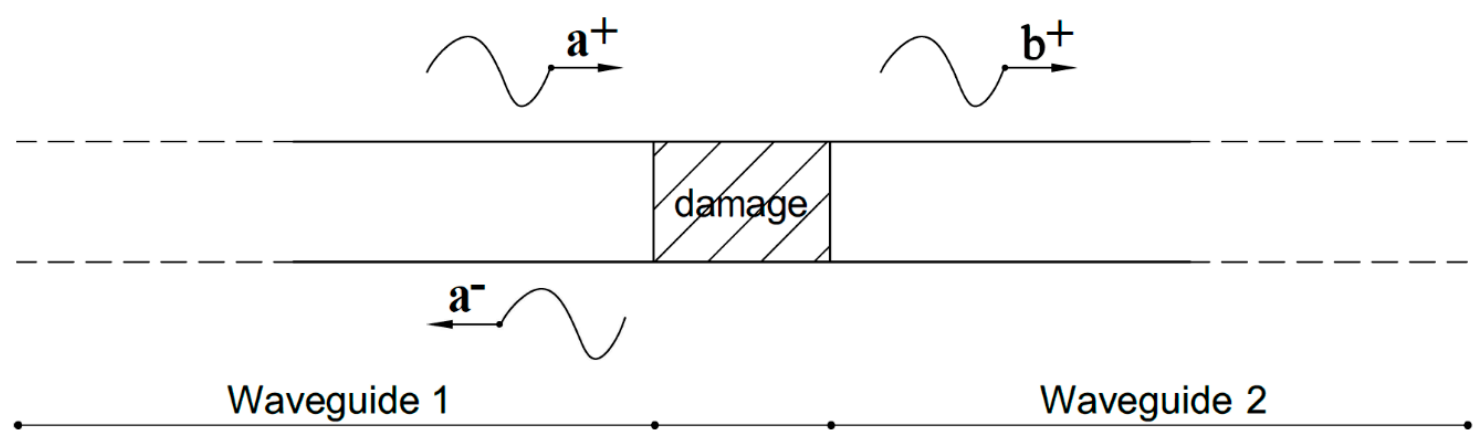

Figure 3. Incident, reflected and transmitted waves in an infinite beam with damage. 


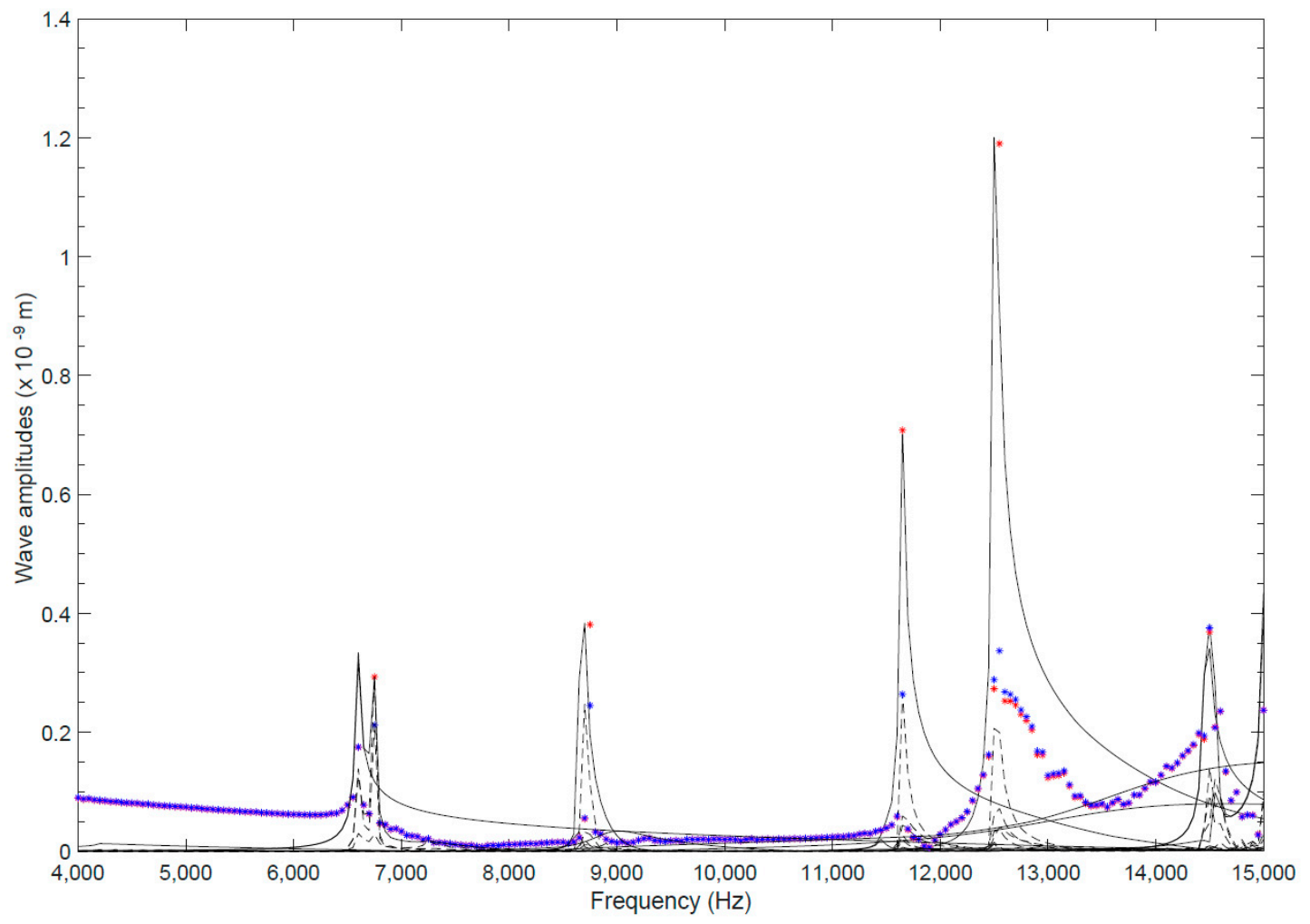

Figure 4. Wave amplitudes of lightly attenuated waves in an infinite damaged RC beam. WFE solution $(-)$, two-wave WAD estimation using $k_{s}=0.1 \mathrm{rad} / \mathrm{m}$ : positive going wave $\left({ }^{*}\right)$, negative going wave $\left(^{*}\right)$.

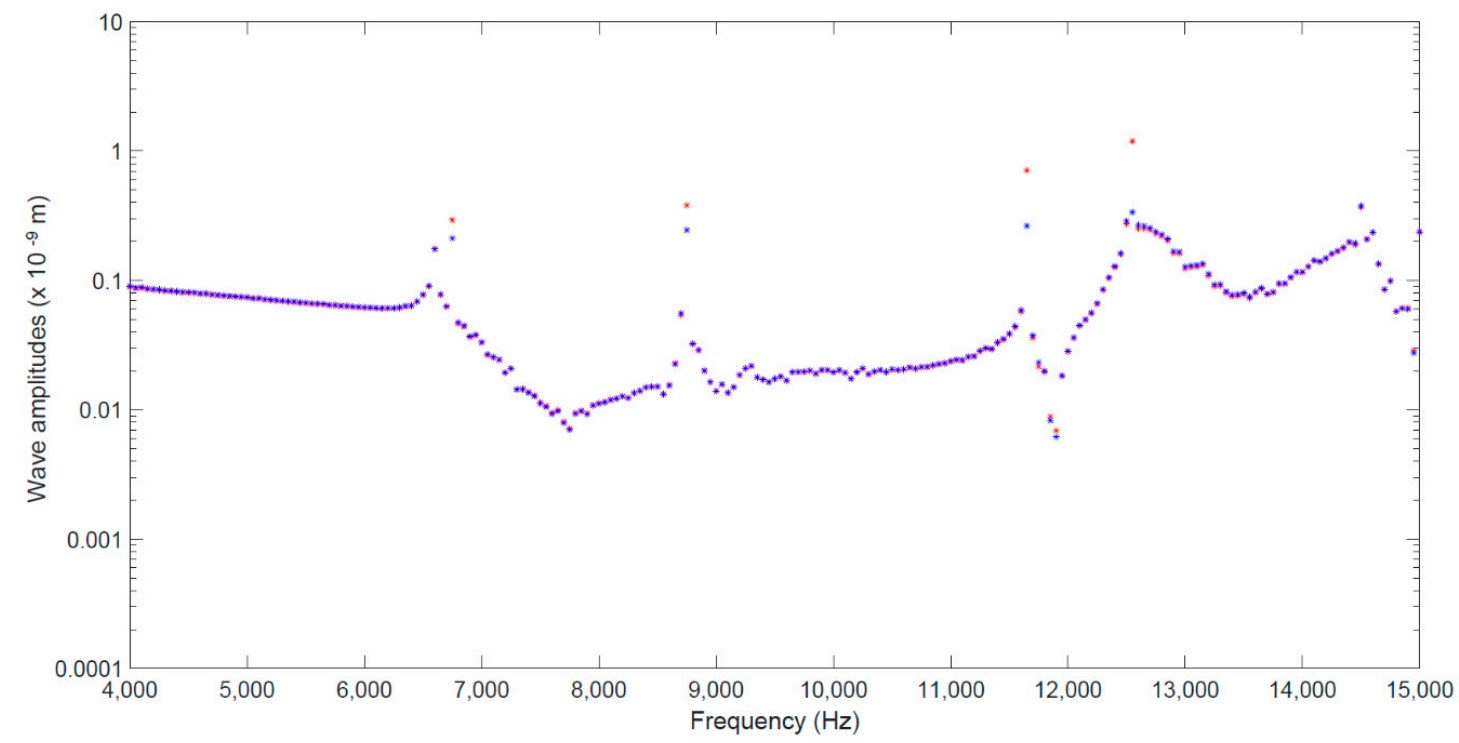

Figure 5. Wave amplitudes in an infinite damaged RC beam obtained by a two-wave WAD estimation using $k_{s}=0.1 \mathrm{rad} / \mathrm{m}$ : positive going wave $\left(^{*}\right)$, negative going wave $\left(^{*}\right)$. 


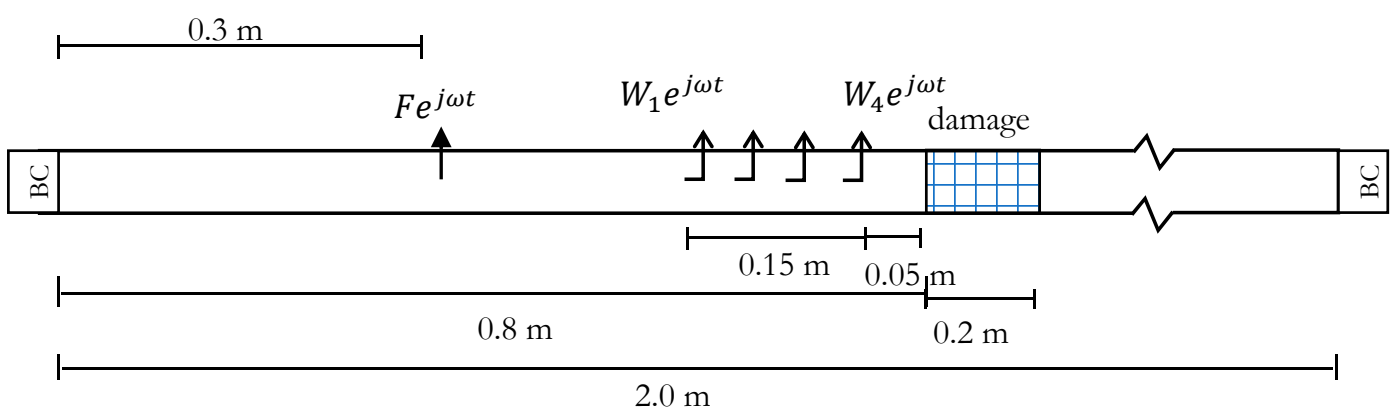

Figure 6. Schematic of a finite RC beam with perfectly reflecting boundaries and a short, damaged section (not to scale) showing the simulated measurement array.
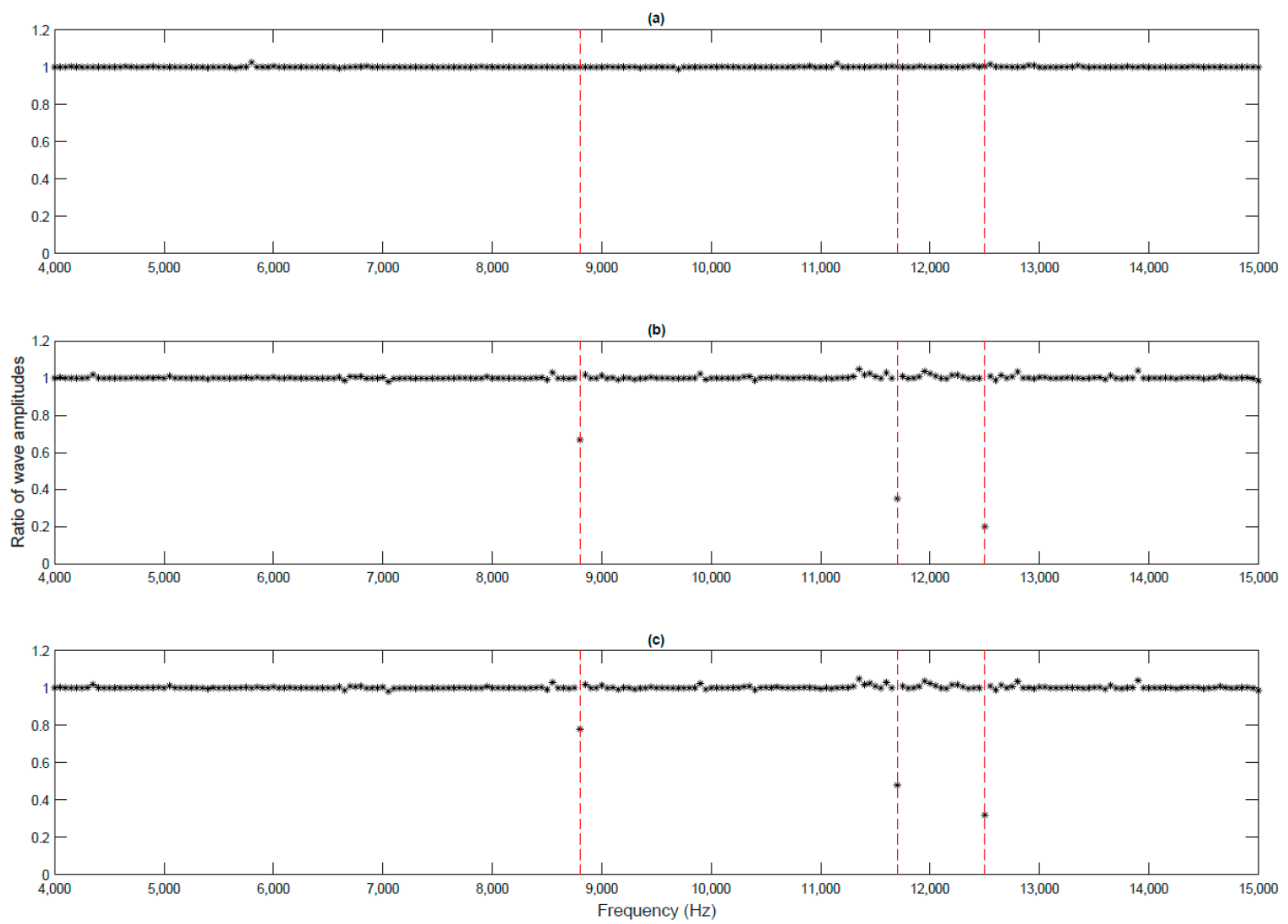

Figure 7. The ratio of estimated negative to positive wave amplitudes for damaged and undamaged RC beams with perfectly reflecting boundaries. (a): undamaged RC beam, (b): 40\% damaged RC beam, (c): $60 \%$ damaged RC beam. Cut-on frequencies (- - ).

Table 2. Reflection coefficients at wave cut-on from a two-wave WAD estimation compared with true values from the infinite beam model.

\begin{tabular}{ccccccc}
\hline Quantity & \multicolumn{2}{c}{$\mathbf{4 0} \%$ Damage in One Rebar } & \multicolumn{3}{c}{$\mathbf{6 0 \%}$ Damage in One Rebar } \\
\hline Cut-on frequency (Hz) & 8800 & 11,700 & 12,500 & 8800 & 11,700 & 12,500 \\
Two-wave WAD estimate & 0.67 & 0.35 & 0.2 & 0.78 & 0.48 & 0.32 \\
$\begin{array}{c}\text { True reflection coefficient } \\
\text { from WFE model }\end{array}$ & 0.72 & 0.4 & 0.21 & 0.80 & 0.53 & 0.30 \\
\hline
\end{tabular}

Figure 8 shows the variation in the estimated reflection coefficient for the three cut-on frequencies as the reduction in diameter of the rebar is varied from $5 \%$ to $100 \%$. Counter to most damage metrics, 
the lowest reflection coefficients, which are most distinguishable from the undamaged case, occur for low levels of damage.

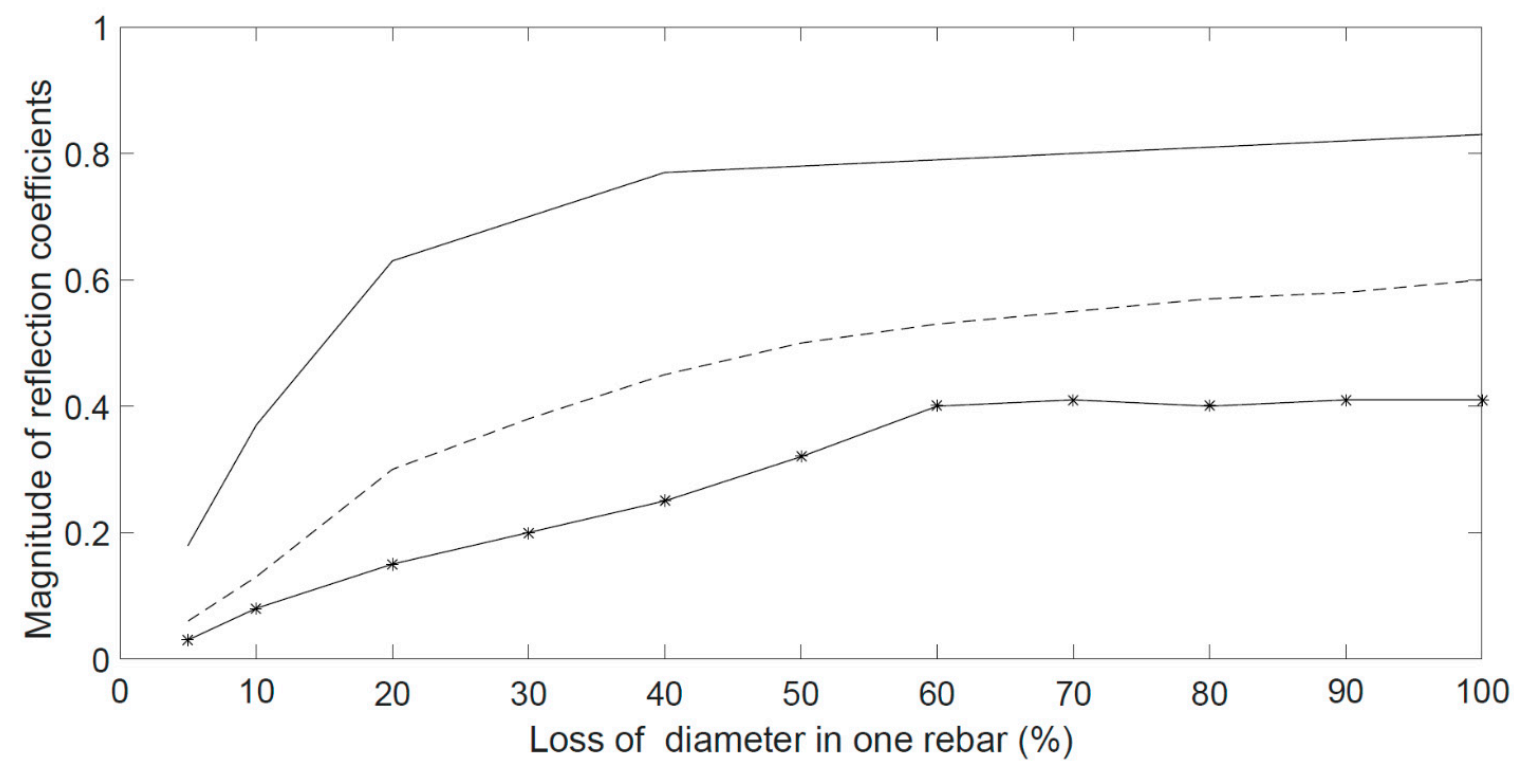

Figure 8. Estimated reflection coefficient at cut-on as a function of loss of diameter in one rebar. $\left(8700 \mathrm{~Hz}-, 11,700 \mathrm{~Hz}-, 12,500 \mathrm{~Hz}_{-}^{-}-\right.$).

\section{Experimental Validation}

Three $2 \mathrm{~m}$ long concrete beams were manufactured, each with four grade 60 steel horizontal rebars, as shown in Figure 9, and vertical stirrups were spaced at $20 \mathrm{~cm}$ intervals. Intact $25 \mathrm{~mm}$ diameter rebars were used in one of the beams. In the other two beams one of the rebars was reduced in diameter to $16 \mathrm{~mm}$ and $10 \mathrm{~mm}$, respectively, over a length of $200 \mathrm{~mm}$ at approximately their midspan, see Figure 10 . The horizontal and vertical reinforcements were tied together using steel fibres as shown in Figure 11. Each beam, weighing about $300 \mathrm{~kg}$, was supported on roller supports as shown in Figure 12.

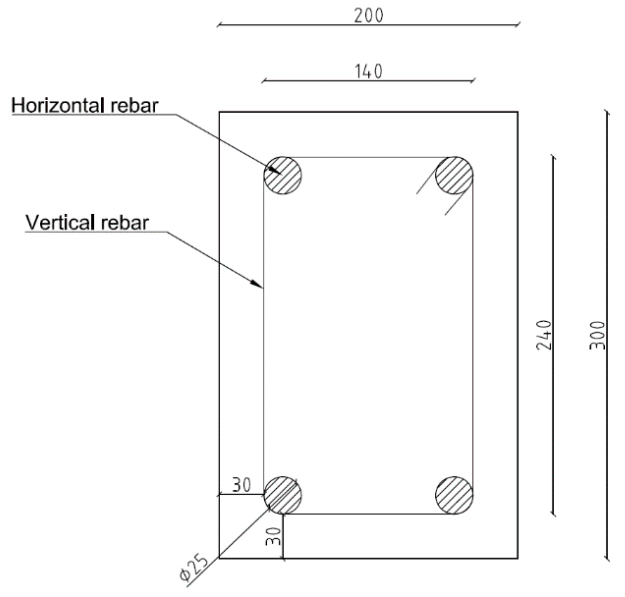

Figure 9. Drawing of RC beam cross-section showing steel reinforcements. Units are in mm.

The concrete mix was composed of the following materials: high-strength cement, water, sand and $10 \mathrm{~mm}$ aggregate. The proportions of each component were first defined by volume, and then by using the proper densities their masses computed for each batch. The mix proportions were selected to be 1:1 by weight for cement and sand. After forming and curing, cylindrical concrete samples were crushed 
to identify the concrete's compressive strength $f_{c}^{\prime}$. The average strength was found to be $70 \mathrm{MPa}$. One can associate the concrete's compressive strength $f_{\mathcal{c}}^{\prime}$ to its Young's Modulus $E_{\mathcal{c}}$ in MPa [19]:

$$
E_{c}=4700 \sqrt{f_{c}^{\prime}}
$$

The associated Young's modulus of concrete at 28 days was found to be approximately 38.9 GPa.

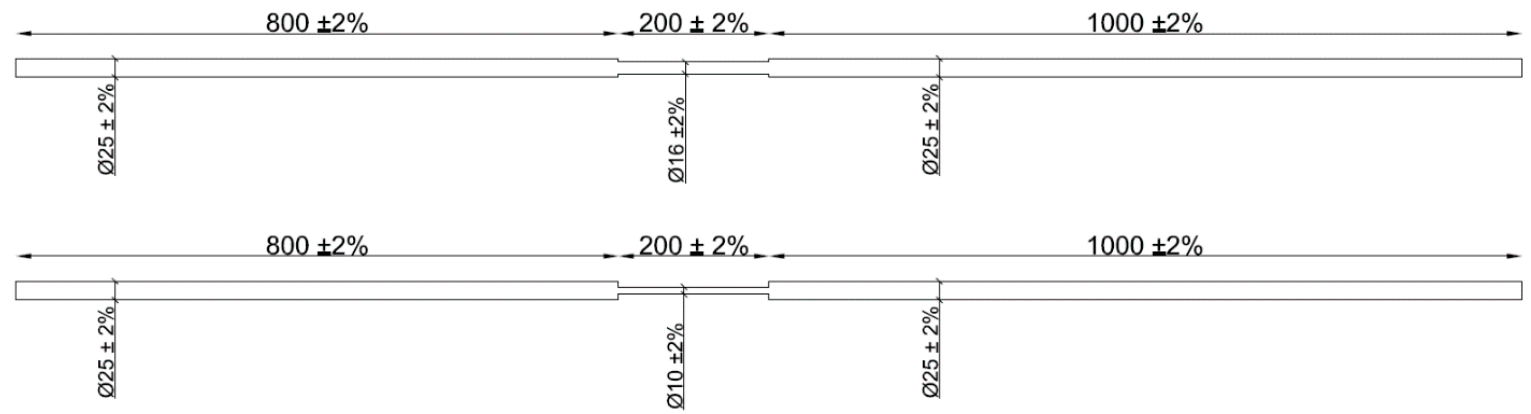

Figure 10. Details of steel reinforcements with localised loss of diameter. Units are in mm.

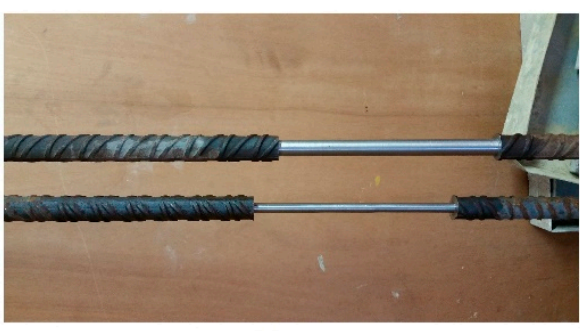

(a)

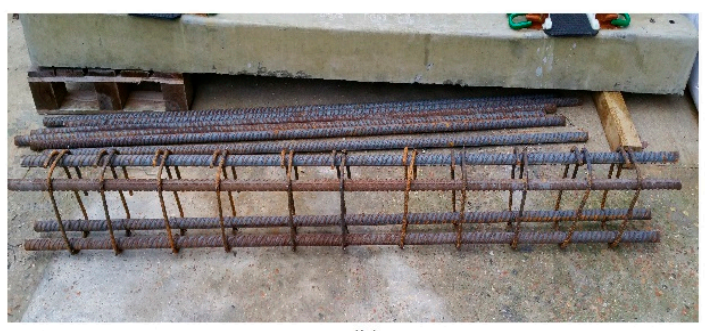

(b)

Figure 11. Images of (a) reduced steel reinforcement bars, (b) assembly of horizontal and vertical steel reinforcements.

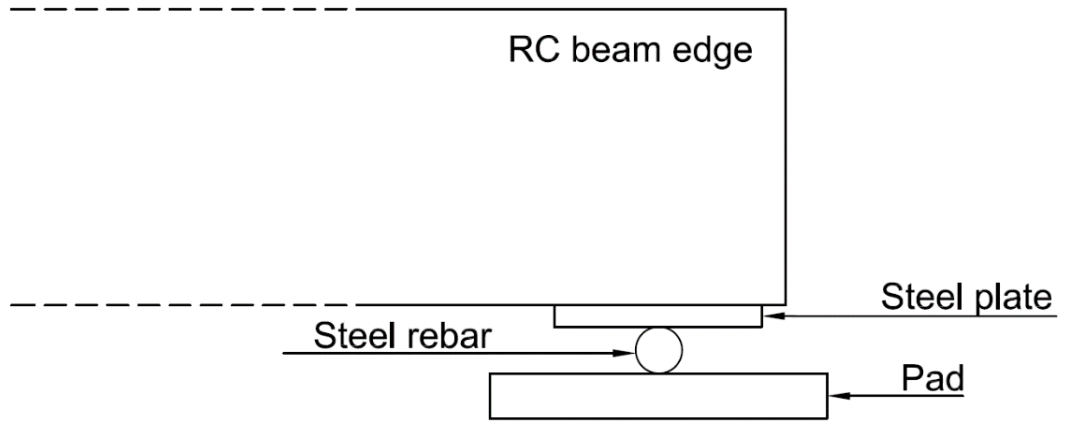

Figure 12. Realisation of experimental boundary conditions.

The concrete covered the whole length of the bar including the region of rebar diameter reduction. Therefore, complete bonding between the steel and surrounded concrete was achieved. A mechanical vibrator was used, after each mix was poured, to homogenise the concrete in the formwork. Without vibration, air bubbles can be entrained in the concrete and a non-homogeneous texture to the beam produced. Excessive vibration leads to separation of the aggregate and mortar. For these reasons, an intermediate period of vibration was employed to ensure proper mixing of the new and old placement of concrete. Once the level of concrete was achieved in the formwork, the concrete was levelled with a small darby tool to ensure that the surface of the concrete was smooth with no surface aggregate or voids. The latter causes local attenuation of the signal. In addition, concrete sealer was applied at the top concrete surface to maintain good bonding with the accelerometers that were mounted with wax. 
Accelerance FRFs were measured using a two-channel instrumented hammer test procedure. The excitation and response positions in the experiment were exactly as for the simulation, i.e., on the top surface of the concrete beam and as shown in Figure 6, except that measurements were performed reciprocally; an accelerometer (model PCB352C22) was fixed at the reference position and the hammer (model PCB086C03) was roved to each array position in turn. Acceleration and force waveforms were acquired using a Data Physics Quattro spectrum analyser. A frequency range of $15 \mathrm{kHz}$ was chosen, over which the structure could be adequately excited by the hammer. A frequency resolution of $10 \mathrm{~Hz}$ ensured complete capture of the transient responses. Figure 13 shows the magnitude of a typical FRF, converted to receptance, where data points with a coherence of less than 0.9 are omitted from the graph and subsequent post-processing.

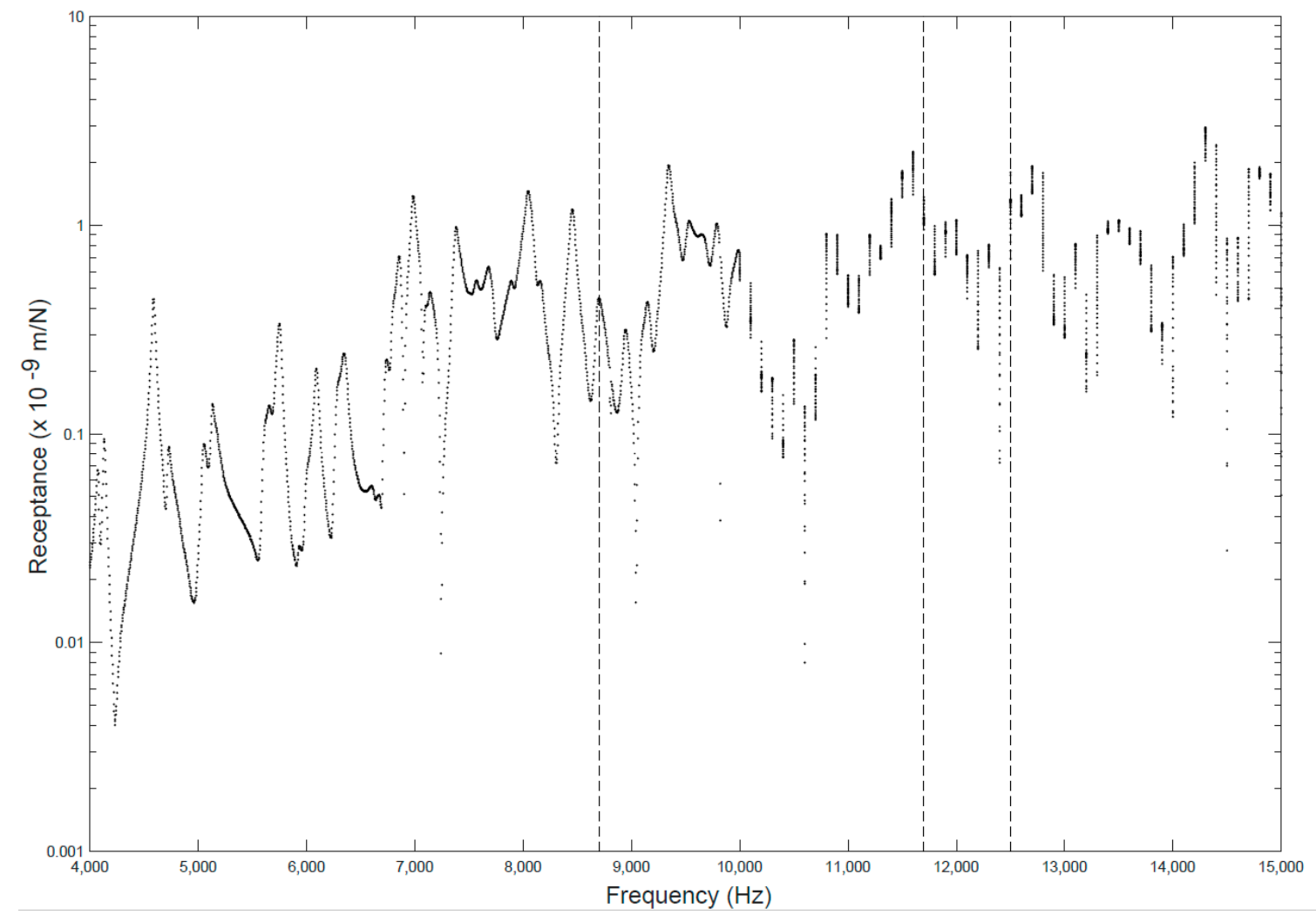

Figure 13. Typical measured receptance from the experimental RC beam. Only data points for which the coherence is greater than 0.9 are shown. Dashed lines are associated with the cut-on frequencies.

A two-wave WAD estimation was used to estimate the reflected and incident wave amplitudes at all retained frequency points. Figure 14 shows their ratio for the undamaged and two-damaged beams. The cut-on frequencies obtained from the tuned model are also shown although no prior knowledge of these, or any aspect of the model, is required by the damage-detection technique. The frequency resolution of the measured data is sufficient to capture clearly visible dips at, or close to, the predicted cut-on frequencies for the damaged beams but not the undamaged beam. Although not clear from the experimental data, less severe damage can be expected to result in narrower dips thus requiring a finer frequency resolution. The slight discrepancy in Figure $14 \mathrm{~b}$ between the minima and the cut-on frequencies is attributable to variability between concrete batches. Away from cut-on, the ratio is unity, as for the simulated study in Section 2, despite the possible occurrence of wave mode conversion at the boundaries in the experimental realisation. The dips are lower in the case of the less damaged beam, as observed in the simulations. The reflection coefficient estimates at cut-on are listed in Table 3; these are in close agreement with true values from the infinite WFE model, also shown. 

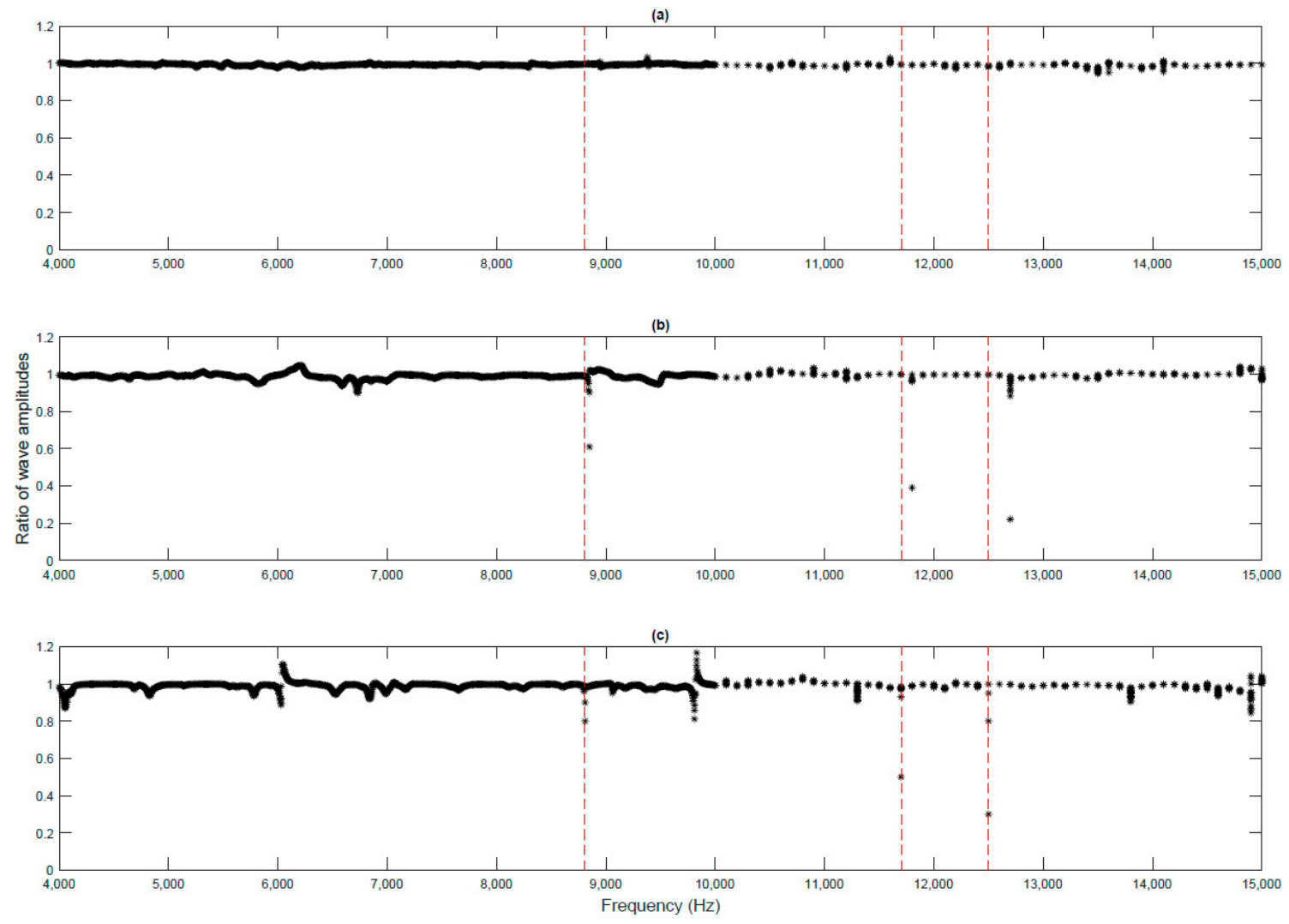

Figure 14. Ratio of estimated reflected to incident wave amplitudes for damaged and undamaged experimental RC beams. (a): undamaged RC beam, (b): $40 \%$ loss of rebar diameter, (c): $60 \%$ loss of rebar diameter. Cut-on frequencies $(---)$.

Table 3. Measured reflection coefficients at wave cut-on from two-wave WAD estimation based on measured frequency response functions (FRFs). Values from the WFE model, with different boundary conditions, are reported for qualitative comparison.

\begin{tabular}{ccccccc}
\hline Quantity & \multicolumn{4}{c}{$\mathbf{4 0} \%$ Damage of One Rebar } & \multicolumn{3}{c}{$\mathbf{6 0 \%}$ Damage of One Rebar } \\
\hline Cut-on frequency $(\mathrm{Hz})$ & 8800 & 11,700 & 12,500 & 8800 & 11,700 & 12,500 \\
\hline $\begin{array}{c}\text { Reflection coefficient from } \\
\text { two-wave WAD estimate } \\
\text { using experimental data }\end{array}$ & 0.60 & 0.39 & 0.22 & 0.80 & 0.50 & 0.30 \\
\hline $\begin{array}{c}\text { True reflection coefficient } \\
\text { from WFE model }\end{array}$ & 0.72 & 0.40 & 0.21 & 0.80 & 0.53 & 0.30 \\
\hline
\end{tabular}

\section{Conclusions}

A technique has been presented for non-invasive inspection of steel reinforcements in concrete beams by observing reflected waves from a localised discontinuity. Reflections are immeasurably small at most frequencies but can be appreciable for a new wave cutting on. Restricting one's attention to cut-on frequencies enables reflection coefficients to be estimated simply with no prior knowledge of wavenumber-frequency relations. Furthermore, no detailed knowledge of cut-on frequencies is necessary, since these are identifiable from the reflection coefficient curves if damage exists. No benchmark experimental data are required from a healthy specimen. A qualitatively accurate model of free wave propagation is beneficial to establish the frequency range of interest over which a modest number of evanescent waves can be expected to cut-on, and to inform sensor placement on the cross section. 
The principal benefit of the technique is its ability to detect the existence of damage from remotely positioned, surface-mounted sensors. Preliminary results reported in [17] indicate that, by enlarging the sensor array, the location of the damage along the length of the beam may also be identifiable. However, determining the nature or extent of the damage, or its location within the cross section, e.g., which rebars are damaged, would require the subsequent deployment of an alternative technique such as ultrasonic tomography.

A combination of simulations and measurements have shown the technique to be robust to various ideal boundary conditions. Further work is required to explore its limitations and domain of applicability, in particular:

(i) to assess the case of a concrete beam in a built-up structure where boundaries give rise to only partial reflection and potentially significant wave mode conversion;

(ii) to validate experimentally more realistic damage scenarios such as those achieved by accelerated corrosion;

(iii) to investigate the location of the sensor array in relation to the damage site, and its proximity to boundaries which give rise to evanescent waves;

(iv) to quantify the effective range of the technique in extensive structures given typical wave attenuation rates; and

(v) to assess the robustness of the technique to reflections from multiple or different damage configurations.

The data presented in this paper are available to download at [20].

Author Contributions: Conceptualization, T.W. and N.F.; methodology E.E.M.; software, E.E.M.; validation, E.E.M.; formal analysis, E.E.M.; investigation, E.E.M.; data curation, E.E.M.; writing—original draft preparation, E.E.M. and T.W.; writing-review and editing, E.E.M., T.W. and N.F.; visualisation, E.E.M.; supervision, T.W. and N.F.; project administration, N.F. All authors have read and agreed to the published version of the manuscript.

Funding: This research was funded by the University of Southampton, the Lebanese Association of Scientific Research (LASeR), and Azm and Saade Association.

Conflicts of Interest: The authors declare no conflict of interest.

\section{References}

1. Sharma, A.; Sharma, S.; Sharma, S.; Mukherjee, A. Investigation of deterioration in corroding reinforced concrete beams using active and passive techniques. Constr. Build. Mater. 2018, 161, 555-569. [CrossRef]

2. Sharma, A.; Sharma, S.; Sharma, S.; Mukherjee, A. Monitoring invisible corrosion in concrete using a combination of wave propagation techniques. Cem. Concr. Compos. 2018, 90, 89-99. [CrossRef]

3. Lei, Y.; Zheng, Z.-P. Review of Physical Based Monitoring Techniques for Condition Assessment of Corrosion in Reinforced Concrete. Math. Probl. Eng. 2013, 2013, 1-14. [CrossRef]

4. Miller, T.; Hauser, C.J.; Kundu, T. Nondestructive Inspection of Corrosion and Delamination at the Concrete-Steel Reinforcement Interface. In Proceedings of the ASME International Mechanical Engineering Congress and Exposition, New Orleans, LA, USA, 17-22 November 2002; pp. 121-128.

5. Li, D.; Ruan, T.; Yuan, J. Inspection of reinforced concrete interface delamination using ultrasonic guided wave non-destructive test technique. Sci. China Ser. E Technol. Sci. 2012, 55, 2893-2901. [CrossRef]

6. Sriramadasu, R.C.; Lu, Y.; Banerjee, S. Identification of incipient pitting corrosion in reinforced concrete structures using guided waves and piezoelectric wafer transducers. Struct. Health Monit. 2018, 18, 164-171. [CrossRef]

7. Sharma, S.; Mukherjee, A. Longitudinal Guided Waves for Monitoring Chloride Corrosion in Reinforcing Bars in Concrete. Struct. Health Monit. 2010, 9, 555-567. [CrossRef]

8. Zheng, Z.; Lei, Y.; Xue, X. Numerical Simulation of Monitoring Corrosion in Reinforced Concrete Based on Ultrasonic Guided Waves. Sci. World J. 2014, 2014, 752494. [CrossRef] [PubMed]

9. Miller, T.H.; Kundu, T.; Huang, J.; Grill, J.Y. A new guided wave-based technique for corrosion monitoring in reinforced concrete. Struct. Health Monit. 2012, 12, 35-47. [CrossRef] 
10. Amjad, U.; Yadav, S.K.; Kundu, T. Detection and quantification of diameter reduction due to corrosion in reinforcing steel bars. Struct. Health Monit. 2015, 14, 532-543. [CrossRef]

11. Majhi, S.; Mukherjee, A.; George, N.V.; Uy, B. Corrosion detection in steel bar: A time-frequency approach. NDT E Int. 2019, 107, 102150. [CrossRef]

12. Zima, B.; Kędra, R. Reference-free determination of debonding length in reinforced concrete beams using guided wave propagation. Constr. Build. Mater. 2019, 207, 291-303. [CrossRef]

13. Climent, M.-Á.; Miró, M.; Carbajo, J.; Poveda-Martínez, P.; Almenar, G.D.V.; Ramis, J. Use of Non-Linear Ultrasonic Techniques to Detect Cracks Due to Steel Corrosion in Reinforced Concrete Structures. Materials 2019, 12, 813. [CrossRef] [PubMed]

14. Zielińska, M.; Rucka, M. Detection of debonding in reinforced concrete beams using ultrasonic transmission tomography and hybrid ray tracing technique. Constr. Build. Mater. 2020, 262, 120104. [CrossRef]

15. El Masri, E.; Ferguson, N.; Waters, T. Wave propagation and scattering in reinforced concrete beams. J. Acoust. Soc. Am. 2019, 146, 3283-3294. [CrossRef] [PubMed]

16. Halkyard, C.; Mace, B.R. Structural intensity in beams-waves, transducer systems and the conditioning problem. J. Sound Vib. 1995, 185, 279-298. [CrossRef]

17. El Masri, E. Wave Propagation in Reinforced Concrete Beams with Application to Non-Destructive Testing. Ph.D. Thesis, University of Southampton, Hampshire, UK, 2018.

18. Renno, J.M.; Manconi, E.; Mace, B.R. A Finite Element Method for Modelling Waves in Laminated Structures. Adv. Struct. Eng. 2013, 16, 61-75. [CrossRef]

19. American Concrete Institute (ACI). Building Code Requirements for Structural Concrete; ACI 318-95; ACI: Farmington Hills, MI, USA, 1995.

20. El Masri, E. Data Set for Journal Paper “Guided Wave Inspection of Bars in Reinforced Concrete Beams Using Surface Mounted Vibration Sensors"; University of Southampton: Hampshire, UK, 2020. [CrossRef]

(C) 2020 by the authors. Licensee MDPI, Basel, Switzerland. This article is an open access article distributed under the terms and conditions of the Creative Commons Attribution (CC BY) license (http://creativecommons.org/licenses/by/4.0/). 